\title{
Las familias biológicas en el proceso de adopción de un menor: características y rasgos sociales
}

\author{
Ana Chacón Martínez \\ Universidad de Murcia
}

\section{Resumen}

Se trata de visibilizar y mostrar las características de las familias biológicas de niños y niñas que son adoptados en la Comunidad Autónoma de la Región de Murcia entre el periodo 1987-2007. Para constatar estos datos se ha realizado un estudio exhaustivo de un total de 29 expedientes de adopción facilitados por la Dirección General de Familias, así como 4 entrevistas a padres adoptantes. Constituyen una muestra y un ejemplo significativo de las características y rasgos sociales de las familias biológicas. A través de estudios de caso reflexionamos sobre si éstas han sido excluidas del proceso de adopción por la caracterización que se desprende de ellas, al estar enmarcadas en contextos problemáticos graves que acentúan el riesgo que supone para los menores vivir en esas familias.

Palabras clave

Padres biológicos; interés del menor; Región de Murcia; siglos XX-XXI

Códigos JEL: K36, J13, R28, H31

\author{
BIOLOGICAL FAMILIES IN THE PROCESS OF ADOPTION OF A \\ MINOR: CHARACTERISTICS AND SOCIAL TRAITS
}

Abstract

It is about making visible and showing the characteristics of the biological families of the boys and girls who are adopted in the Autonomous Community of the Region of Murcia between the period 1987-2007. To verify these data, an exhaustive study of a total of 29 adoption files provided by the General Directorate of Families, as well as 4 interviews with adoptive parents. They constitute a sample and a significant example of the characteristics and social traits of biological families. Through case studies, we reflect on whether they have been excluded from the adoption process due to the characterization that emerges from them, as they are framed in serious problematic contexts that accentuate the risk that the minors entail living in these families.

\section{Keywords}

Biological parents; interest of the minor; Region of Murcia; $\mathrm{XX}-\mathrm{XXI}$ centuries

JEL codes: K36, J13, R28, H31

Fecha de recepción del original: 10 de agosto de 2021; versión definitiva: 31 de octubre de 2021.

Ana Chacón Martínez. Departamento de Trabajo Social y Servicios Sociales, Universidad de Murcia. Campus de Espinardo, 30.100, Murcia. Tel.: +34 633410340; E-mail: ana.chacon@um.es; ORCID ID: https://orcid.org/0000-0001-6885-4860. 


\title{
Las familias biológicas en el proceso de adopción de un menor: características y rasgos sociales
}

\author{
Ana Chacón Martínez \\ Universidad de Murcia
}

\section{Introducción}

Las infancias desoladas que Charles Dickens ${ }^{1}$ presentaba en sus historias literarias, no eran solo producto de un tiempo contemporáneo, resultado de la imperiosa industrialización del Siglo XIX. Antes de todo eso, entonces $y$ ahora, las realidades crueles que han afectado al colectivo infantil son más graves de lo que puede presentar cualquier ficción. La orfandad, o cualquier otra condición o situación de malvivir, que lleva implícita la pobreza, la marginación o la exclusión social, acucia más gravemente a los menores de edad, cuya peor baza es la indefensión, propia de la falta de experiencia que requiere haber vivido el tiempo necesario para adquirirla.

Si son contemporáneos, sin embargo, la toma de conciencia y los cambios culturales que se han producido en la sociedad, en cuanto a la posibilidad de solucionar las situaciones de desamparo infantil, porque romper con la tradición y la creencia de que los lazos de sangre son el único vínculo aceptable y reconocible en la estructura familiar, ha llevado siglos. Todavía en el siglo XIX se "pagaba" o "cobraba" por "acoger" a niños y niñas en el seno familiar, casi siempre con la finalidad de que ejecutaran trabajos de cualquier índole a cambio de casa y comida. A las alturas del siglo XXI, no puede afirmarse que se hayan desarticulado todos los perjuicios al respecto, pues no dejan de ser demasiado recientes la Declaración Universal de los Derechos Humanos (1948), y la Declaración de los Derechos del Niño (1959). A partir de estas fechas gran parte de la sociedad occidental, junto con los cambios generados en los modelos de familia y en el Estado de Bienestar Social, ha venido reconociendo, aceptando y practicando la adopción como una forma normalizada de constituir familias.

\footnotetext{
1 En plena revolución industrial en Inglaterra, Charles Dickens (Inglaterra, 18121870) denunció a través de sus obras literarias, publicadas por entregas en la prensa, las situaciones de pobreza y exclusión social a las que daba lugar las desigualdades sociales ocasionadas por dicha industrialización, y especialmente de la situación de la infancia, la de los huérfanos y de las instituciones que se encargaban de su cuidado, así lo ponía de manifiesto en obras como: Oliver Twist (1837-1839), Tiempos difíciles (1854) o La pequeña Dorrit (1855-1857), entre otras.
}

No obstante, la necesidad de aunar los diversos agentes que intervienen en la adopción: desde el Estado y su ámbito jurídico administrativo en todas sus dimensiones, a las familias biológicas y adoptivas, así como a los propios menores adoptados -o que supone una implicación personal en todos los aspectos que atañen al ser humano- han hecho de la adopción una cuestión de complejo entramado, todavía no lo suficientemente dilucidado, por la certera existencia de una amplia problemática que necesita de investigaciones profundas que las pongan en evidencia para poder buscar soluciones precisas. Soluciones que deben dirigirse, especialmente, a evitar daños emocionales, psicosociales, e incluso materiales que, entre otros muchos, lleva consigo el proceso de vinculación y desvinculación individual, familiar y extrafamiliar que supone la adopción.

Por tanto, desatar y comenzar a leer los, normalmente muy voluminosos, legajos depositados en el Archivo Histórico General de la Región de Murcia (AHGRM); o aquellos que, todavía, no han sido cerrados, definitivamente, y se encuentran en las dependencias administrativas de la Consejería de Política Social e Igualdad de Oportunidades donde se han iniciado, es algo parecido a abrir una ventana $a$ un pasado reciente que se encuentra vivo y en plena evolución. Los protagonistas de estas historias de vida han dejado toda una amplia serie de testimonios, signos, evidencias e indicaciones cuya fiabilidad está confirmada y contrastada por los informes y expedientes de los profesionales responsables de su cuidado, atención y gestión. En nuestro estudio, por tanto, nos vamos a referir a las familias biológicas, tanto al padre como a la madre, para visibilizarlas en el complejo entramado que supone un proceso de adopción de un niño o niña, el cual es retirado de su familia natural o/y entregado de forma voluntaria a la familia adoptiva.

Nos encontramos ante, en palabras de Arlette Farge (1991: 25): trozos de verdad o pedazos de vida que producen una unión entre el pasado y el presente. Un pasado del que nuestro análisis cuantitativo y cualitativo deducirá tendencias y resultados, que permitirán obtener conclusiones para confirmar o bien proponer y mejorar políticas públicas sobre el conjunto de los protagonistas y su integración e inclusión en el sistema social. Pero este pasado no puede en ningún momento, situación, ni coyuntura, desprenderse o separarse del presente. Precisamente, las 
enseñanzas que se pueden concluir de nuestro estudio forman parte de propuestas de mejora que incidirán sobre el futuro de quienes son adoptados e inician una fase descrita en su trayectoria de vida.

La finalidad y el propósito de este trabajo será analizar las características psicosociales y los rasgos de la familia biológica, la actividad profesional, la edad de los padres y madres biológicos al primer hijo tenido, así como el origen y estado civil de los mismos. Igualmente, y a modo de ejemplo, estudiaremos algunos casos sobre intentos de integración del menor en su familia biológica.

\section{Planteamiento y objetivos}

Si realizamos una aproximación a la existencia de literatura científica relativa a las familias biológicas en España, encontramos escasos textos que hagan referencia a estudios exhaustivos sobre ellas. En la actualidad, el principio de prioridad en la familia natural, recogido en el art. 172.4 del Código Civil: "se buscará siempre el interés del menor y se procurará, cuando no sea contrario a ese interés, su reinserción en la propia familia y que la guarda de los hermanos se confíe a una misma institución o persona"2, es uno de los factores básicos en el marco de las actuaciones de protección del menor. Por tanto, resulta cuando menos necesario reflexionar, pese a las pocas referencias bibliográficas sobre la familia biológica, sobre los menores que son retirados o entregados voluntariamente a la Administración por encontrarse desprotegidos en sus familias de origen. Si estudiamos más en profundidad a la familia natural podremos adoptar mejores políticas públicas que ayuden a reintegrar al menor en su propia familia. De esta manera, cumpliremos con el mandato del legislador (artículo 172.4 Código Civil); es decir, establecer en todas las actuaciones relacionadas con niños y las niñas el principio del interés superior del menory estudiar si lo más conveniente para los menores es reintegrarlo en su familia de origen, o, por el contrario, comenzar el proceso de acogimiento y/o adopción.

Se construye, por tanto, un marco jurídico de protección que vincula a todos los poderes públicos y que estructura el derecho a la protección de la infancia vigente en España desde siglos atrás; reflejado, progresivamente, en distintas leyes a lo largo de la historia (Chacón Martínez, 2019: 40). Incluso el legislador va más allá, aprobando recientemente la Ley Orgánica 8/2021, de 4 de junio, de protección integral a la infancia y la adolescencia frente a la violencia (BOE no 134, 05/06/2021). Esta Ley atiende al derecho de los niños, niñas y adolescentes de no ser objeto de ninguna forma de violencia, estableciendo medidas de protección, detección precoz, asistencia, reintegración de derechos vulnerados y recuperación de la víctima.

\footnotetext{
${ }^{2}$ Artículo 172.4 Código Civil.
}

Señalaremos algunos ejemplos que nos permiten acercarnos más a la realidad social de estas familias. En un sugerente y documentado estudio sobre: "perspectivas y tendencias del acogimiento familiar en Cataluña", Pere Amorós y Nuria Fuentes (Marre y Bestard, 2004: 20) señalan las características de las familias biológicas que en Cataluña, a lo largo del período 1992-2000, obligaron a la Administración a recoger a sus hijos para ser entregados en acogimiento. Escasos recursos económicos, presencia de drogodependientes, malos tratos $u$ otras situaciones problemáticas, muestran una realidad que se confirma con las características psicosociales de las familias biológicas en el caso de Murcia durante 1987-2007, como veremos más adelante.

También, algunos autores, según señala Milagros Fernández (2004), encuentran:

Un altísimo grado de desestructuración familiar, el $90 \%$ de padres no tiene cualificación laboral, el $30 \%$ de las madres se dedica a la prostitución, y más de un $40 \%$ de los padres y un $25 \%$ de las madres han tenido problemas con la ley. El $49 \%$ de los padres y el $60 \%$ de las madres son toxicómanos. La relación padres-hijos, una vez iniciado el acogimiento, es poco frecuente $y$, cuando existe, suele ser conflictiva (Fernández, 2004: 26).

Más recientemente, también Reyes y Cantera Rey (2021) analizaron la familia biológica en el acogimiento familiar y las prácticas de exclusión de las que han sido parte en los proyectos terapéuticos/ asistenciales que realizan los trabajadores sociales, y su logro o no en los cambios necesarios para la reunificación de los menores en los sistemas de origen, es decir, en sus familias naturales.

Mayor del Hoyo (2020), aunque se refiera a aspectos mucho más formales, presenta un trabajo centrado en el asentimiento de la madre biológica, así como la forma de emisión, y la capacidad de la madre para prestarlo. Reflexiona igualmente sobre una posible limitación de la autonomía de la voluntad y una propuesta de lege ferenda para garantizar un equilibrio entre las necesidades de los menores y el libre ejercicio de la libertad de la mujer.

En una investigación realizada sobre los procesos de adopción entre 1977 y 1992 en la Santa Casa de la Misericordia de Lisboa (Salvatierra, 2003: 39), se registraron una serie de conclusiones respecto a la madres biológicas; cuáles fueron los motivos de la adopción (si hubo consentimiento previo, abandono o fue por mandato judicial), el estado civil de la madre biológica (desconocido, casada, viuda, divorciada, separada o soltera), o la edad de la madre al dar en adopción a sus hijos.

El objetivo general de esta investigación, y su denominación concreta, es: estudiar, conocer y explicar los aspectos sociales y de origen de la familia natural de los adoptados. Se establecen las condiciones sociales y de origen, tanto de la madre biológica como del padre biológico, según una serie de variables cuantitativas y cualitativas, como son: edad, origen, estado civil, formación académica o problemática familiar.

Esta información se explica a través de 29 expedientes estudiados y analizados, pretendiendo conseguir con este 
Tabla 1. Datos de las familias adoptantes entrevistadas

\begin{tabular}{|l|c|c|c|c|}
\hline \multicolumn{1}{|c|}{ Entrevistado/a } & 1 (E.M.G.) & 2 (J.A.) & 3 (C.G.G.) & (E.S.L.) \\
\hline Sexo & Mujer & Hombre & Mujer & Mujer \\
\hline Fecha de la entrevista & $09 / 02 / 2015$ & $20 / 03 / 2015$ & $29 / 05 / 2015$ & $23 / 06 / 2015$ \\
\hline Lugar de la entrevista & $\begin{array}{c}\text { Residencia de la entre- } \\
\text { vistada }\end{array}$ & Lugar de trabajo & Lugar de trabajo & $\begin{array}{c}\text { Residencia de la } \\
\text { entrevistada }\end{array}$ \\
\hline $\begin{array}{l}\text { Fecha de Nacimiento/edad } \\
\text { a la fecha de la entrevista }\end{array}$ & $1972 / 43$ años & $1959 / 56$ años & $1968 / 47$ años & $1970 / 45$ años \\
\hline Lugar de residencia & Murcia & La Alcayna (Murcia) & Murcia & Murcia \\
\hline Nivel de estudios & Bachillerato & Universitario & Universitario & Bachillerato \\
\hline Profesión & Desempleada & Docente & Docente & Empleada de hogar \\
\hline Estado civil & Soltera & Casado & Casada & Casada \\
\hline
\end{tabular}

objetivo acercarnos a la realidad social de estas madres y padres que, por factores externos o de cualquier otra índole, deciden entregar a sus hijos e hijas en adopción. También de los casos en donde la entidad pública debe de actuar y asumir la tutela de los menores, quitándosela a los progenitores biológicos.

En consecuencia, pretendemos contribuir al conocimiento, caracterización y casuística de las familias biológicas mediante el ejemplo de la Región de Murcia. Y de esta forma elaborar posibles propuestas que avancen en el sentido y orientación marcada por el legislador. Sin embargo, la realidad económico-social de España y los ejemplos que podemos aportar, demuestran las graves dificultades y problemas para conseguir el objetivo jurídico señalado en el ya citado art. 172.4 del Código Civil. Ello explica el rechazo de las familias adoptantes a mantener contacto con las familias biológicas, aunque esta palabra encierra una falsa realidad y un eufemismo pues, como se demuestra a continuación, no existe, normalmente, familia, sino madres biológicas.

\section{Metodologia y fuentes}

La investigación que aquí se presenta está basada en un estudio cuantitativo en el que se valoran y examinan aquellos aspectos susceptibles de cuantificación que contienen los expedientes de adopción; y un estudio cualitativo a partir de los discursos de los actores sociales que participan en el proceso.

Los principales documentos en los que se ha basado nuestro análisis, y que conforman la estructura de este tra- bajo, proceden de dos archivos: Archivo Histórico General de la Región de Murcia (en adelante AHGRM) (18 expedientes de adopción, que corresponden al periodo 1987-2000) y Registro General del Servicio de Adopciones (11 expedientes de adopción, que corresponden al periodo 19992007) de la Comunidad Autónoma de la Región de Murcia (en adelante, CARM).

Las principales fuentes revisadas, además de los 29 expedientes de adopción, han sido datos del Instituto Nacional de Estadística (INE) y 4 Entrevistas a padres adoptivos (realizadas entre febrero y junio de $2015^{3}$ ). Los datos identificativos, referidos a las variables básicas de las personas entrevistadas son los que se recogen en la Tabla 1.

Los criterios de selección de las personas entrevistadas han estado determinados, en el caso de las familias adoptantes, por el conocimiento personal, en un primer momento, que ha llevado al llamado efecto "bola de nieve". Ha permitido contactar con tres parejas, de las cuales uno de los miembros de la pareja se ha prestado a ser entrevistado; y una adoptante monoparental. Se ha realizado una entrevista a cada persona seleccionada durante un tiempo de dos horas, aproximadamente. Todas las personas entrevistadas aceptaron someterse a las mismas.

Los expedientes son designados por la Administración competente en materia del menor, con número/año en curso (por ejemplo: 234/2004), con expresión del nombre del menor o apellidos de la familia natural. Pero, a fin de preservar la identidad y guardar el anonimato de cuantas personas intervienen, hemos sustituido en todo el trabajo

\footnotetext{
3 De las cuatro entrevistas a las familias adoptantes, tres de ellas son parejas heterosexuales y una monoparental. De las tres biparentales conformadas por matrimonios de distinto sexo, han respondido dos madres adoptivas y un padre adoptivo, y en cuanto a la familia monoparental, se trata de una madre adoptiva. Las entrevistas en profundidad de familias adoptantes responden a un diseño semiestructurado de entrevista, con un control medio-abierto a posibles nuevas preguntas--, con un diseño de investigación planificado, un guión de preguntas abiertas y ordenadas, que permite profundizar en el conocimiento de las relaciones entre la familia biológica y la adoptiva.
} 
la signatura original de cada expediente por un número ficticio. La apertura de los expedientes es otra de las complicaciones que presenta el estudio, ya que es compleja la variabilidad de los motivos que dan lugar a la misma ${ }^{4}$.

Tabla 2. Relación de expedientes analizados, 1987-2007

\begin{tabular}{|l|l|r|r|r|l|}
\hline $\begin{array}{c}\text { No atri- } \\
\text { buido de } \\
\text { expedi- } \\
\text { ente }\end{array}$ & $\begin{array}{c}\text { Fecha } \\
\text { inicio }\end{array}$ & $\begin{array}{c}\text { Fecha } \\
\text { final }\end{array}$ & $\begin{array}{c}\text { Admini- } \\
\text { stración }\end{array}$ & $\begin{array}{c}\text { Duración } \\
\text { expe- } \\
\text { diente } \\
\text { (años) }\end{array}$ & $\begin{array}{c}\text { Ubica- } \\
\text { ción para } \\
\text { consulta }\end{array}$ \\
\hline 1 & 1987 & 1989 & CARM & 2 & AHGRM \\
\hline 2 & 1987 & 1992 & CARM & 5 & AHGRM \\
\hline 3 & 1987 & 1991 & CARM & 4 & AHGRM \\
\hline 4 & 1988 & 1993 & CARM & 5 & AHGRM \\
\hline 5 & 1997 & 2004 & CARM & 7 & AHGRM \\
\hline 6 & 1997 & 1998 & CARM & 1 & AHGRM \\
\hline 7 & 1987 & 1999 & CARM & 12 & AHGRM \\
\hline 8 & 1996 & 2000 & CARM & 4 & AHGRM \\
\hline 9 & 1997 & 2008 & CARM & 11 & AHGRM \\
\hline 10 & 1998 & 2001 & CARM & 3 & AHGRM \\
\hline 11 & 1998 & 2004 & CARM & 6 & AHGRM \\
\hline 12 & 1997 & 2004 & CARM & 7 & AHGRM \\
\hline 13 & 1991 & 2007 & CARM & 16 & AHGRM \\
\hline 14 & 1997 & 2005 & CARM & 8 & AHGRM \\
\hline 15 & 1998 & 1998 & CARM & 2 meses & AHGRM \\
\hline 16 & 1998 & 2002 & CARM & 4 & AHGRM \\
\hline 17 & 1998 & 2002 & CARM & 4 & AHGRM \\
\hline 18 & 1994 & 1999 & CARM & 5 & AHGRM \\
\hline 19 & 2002 & 2004 & CARM & 2 & CFIO \\
\hline 20 & 1999 & 2004 & CARM & 5 & CFIO \\
\hline 21 & 1999 & 2004 & CARM & 5 & CFIO \\
\hline 22 & 2000 & 2003 & CARM & 3 & CFIO \\
\hline 23 & 2003 & 2009 & CARM & 6 & CFIO \\
\hline 24 & 2004 & 2006 & CARM & 2 & CFIO \\
\hline 25 & 2006 & 2009 & CARM & 3 & CFIO \\
\hline 26 & 2006 & 2008 & CARM & 2 & CFIO \\
\hline 27 & 2006 & 2007 & CARM & 1 & CFIO \\
\hline 28 & 2008 & 2009 & CARM & 1 & CFIO \\
\hline 29 & 2007 & 2009 & CARM & 2 & CFIO \\
\hline
\end{tabular}

La duración del expediente es muy heterogénea, y difícil de determinar. Por ello, en los casos estudiados se aprecia cómo unos expedientes se resuelven de forma rá-

\footnotetext{
4 Hay expedientes que se abren por la denuncia de una trabajadora social del mal cuidado de los niños (exp.2); una señora que dice tener tres meses a unos niños que le dejó la madre, y no ha vuelto (exp.5 y 8); una madre que comparece diciendo que no puede hacerse cargo de sus hijos (exp.6), o una abuela que no puede hacerse cargo del menor a su cuidado (exp.17). En otras ocasiones, la apertura viene determinada por la Administración Pública o por las Fuerzas y Cuerpos de Seguridad del Estado. Para comprobar fecha de inicio, fecha final, administración, duración del expediente y ubicación para consulta, ver Tabla 1.
}

pida, (son los menos, por ejemplo, exp.15), mientras otros duran algunos años (exp.17); y en algunos casos bastantes años (exp.9), por diferentes causas: porque son varios hijos, por familias muy conflictivas, por oposición de los padres biológicos, o bien presentando recursos ante el juzgado por no estar de acuerdo con las medidas adoptadas por la Administración. El final del expediente llega cuando los menores son dados de baja en la Sección de Protección y Tutela al haber sido acogidos y/o adoptados: porque no ha funcionado el acogimiento y vuelven al Centro de Acogida, o porque han llegado a la mayoría de edad.

Cada expediente consta de todos los escritos, informes y documentos referidos a los protagonistas que intervienen en el proceso de adopción. En nuestro trabajo nos centraremos únicamente en la familia biológica. Los informes referidos a estas familias están hechos por los Departamentos/Servicios de la Consejería encargada de las competencias de Política Social como el ISSORM, o la Secretaría Sectorial de Acción Social. Se incluye también documentación judicial y realizada por otras instituciones: guardia civil, policía, ayuntamientos, centros de menores y hospitales. La información y documentos contenidos en los expedientes sobre la familia biológica se reflejan en la Tabla 3.

Tabla 3. Informes y documentos contenidos en los expedientes de la familia biológica, 1987-2007

\begin{tabular}{|l|}
\hline Informes sociales \\
\hline Informes médicos \\
\hline Informes de orientación \\
\hline Comparecencias de la familia biológica \\
\hline Intervención psicosocial \\
\hline Visitas de la familia biológica al menor \\
\hline Declaración de consentimiento de la familia biológica \\
\hline Informes de valoración \\
\hline Informes de visita domiciliaria \\
\hline Informes de incidencias \\
\hline Declaraciones de la familia biológica \\
\hline Entrevistas \\
\hline Renuncias de la madre biológica \\
\hline
\end{tabular}

El elevado número de informes y documentación de algunos expedientes así como su heterogeneidad y dispersión del contenido, nos ha obligado a sistematizar y ordenar la información de manera adecuada. A partir de toda la información recogida en los expedientes se ha elaborado una base de datos dónde se registran, organizan y clasifican las posibles variables y referencias para poder estudiar y analizar la familia biológica. Esta informatización de los datos nos va a permitir cuantificar todas las variables de estudio de la familia biológica. 
Tabla 4. Factores psicosociales familia biológica. 1987-2007*

\begin{tabular}{|c|c|c|}
\hline \multicolumn{3}{|c|}{ Mujeres } \\
\hline \multicolumn{3}{|c|}{ Comportamiento } \\
\hline & Frecuencia & $\%$ \\
\hline Delincuente & 1 & 3,5 \\
\hline Drogadicta y nómada & 1 & 3,5 \\
\hline $\begin{array}{l}\text { Irresponsables/Despegadas/ } \\
\text { Desentendidas }\end{array}$ & 11 & 39,2 \\
\hline Inestables e inmaduras & 3 & 10,7 \\
\hline Abandono de hijos & 3 & 7,1 \\
\hline Prostitución & 1 & 3,5 \\
\hline Total & 19 & 67,5 \\
\hline \multicolumn{3}{|c|}{ Deficiencias y enfermedades } \\
\hline Retraso mental y alcohólica & 1 & 3,5 \\
\hline Deficientes y desentendidas & 3 & 10,7 \\
\hline Esquizofrenia y marginal & 1 & 3,5 \\
\hline Total & 5 & 17,7 \\
\hline \multicolumn{3}{|c|}{ Otros problemas } \\
\hline Sin papeles & 4 & 14,2 \\
\hline Total & 4 & 14,2 \\
\hline Total General & 28 & 100,0 \\
\hline
\end{tabular}

\begin{tabular}{|l|c|c|}
\hline \multicolumn{3}{|c|}{ Hombres } \\
\hline & Comportamiento \\
\hline & 2 & 5,7 \\
\hline $\begin{array}{l}\text { Delincuente y antece- } \\
\text { dentes penales }\end{array}$ & 3 & 8,5 \\
\hline $\begin{array}{l}\text { Irresponsable/ Maltra- } \\
\text { tador/ Desentendido }\end{array}$ & 1 & 2,8 \\
\hline Conflicto de pareja & 4 & 11,4 \\
\hline Alcohólicos & 10 & 28,4 \\
\hline Total & 2 & 5,7 \\
\hline \multicolumn{2}{|c|}{ Deficiencias y enfermedades } \\
\hline Deficiente mental & 1 & 2,8 \\
\hline Problemas psíquicos & 3 & 8,5 \\
\hline Total & 1 & 2,8 \\
\hline \multicolumn{2}{|c|}{ Otros problemas } \\
\hline Sin papeles & 21 & 60 \\
\hline Desconocidos & 35 & 100,0 \\
\hline Total & 22 & 62,8 \\
\hline \multicolumn{2}{|c|}{ Total General } & 2 \\
\hline
\end{tabular}

$\left(^{*}\right)$ "[...] el número de 28 mujeres y 35 hombres pese a la existencia de 29 expedientes se debe a que, en el caso de las mujeres, dos hermanos se encuentran en diferente expediente, pero es la misma madre; por lo que respecta a los hombres, son 35 porque algunos hijos son de diferentes padres pero de la misma madre. El problema de la promiscuidad y el desconocimiento de la información en el caso de 21 hombres explican estas, aparentes, diferencias en las cifras".

\section{Análisis de los resultados y discusión}

\subsection{La familia biológica: rasgos y características sociales}

Uno de los agentes sociales protagonistas del proceso de adopción son las familias biológicas. Estudiar las condiciones sociales de dichas familias cuyos niños y niñas son adoptados, es enfrentarse a una realidad dura, penosa, casi dramática y muy próxima a la marginación, especialmente en el caso de las madres; más aún cuando sabemos que la mayoría de ellas han pasado por un periodo complicado, difícil y casi estremecedor justo en el instante de dar a luz a sus hijos o después del parto. Esta situación es relatada en los expedientes de adopción cuando la entidad pública intenta reorientar a esa familia biológica y propone medidas para que los menores vuelvan al seno de su familia natural.

Los expedientes nos hablan de madres biológicas con diversidad de problemas personales relacionados con las circunstancias que le rodean. Características propias de la personalidad que han ido adquiriendo y conformando a lo largo del tiempo. Sugiero al lector una mirada atenta sobre las indicaciones y términos con los que los técnicos del servicio de adopción de las entidades públicas califican las características y problemáticas personales de dichas madres. El uso del lenguaje y el análisis de este tipo de discurso pone en relación palabras con realidades sociales. La lectura refleja, claramente, unas formas de vida y situaciones que explican la decisión de la Administración de retirarle los hijos biológicos a estas madres, tal y como señalamos a continuación y se especifica en la Tabla 4.

Los equipos técnicos de la Administración Pública indican y precisan comportamientos como: irresponsabilidad, desentendimiento, inmadurez, y conductas sociales reprobables: alcoholismo, drogadicción ${ }^{5}$, prostitución. Problemas también de origen médico: retraso mental, esquizofrenia o epilepsia. Un ejemplo de comportamiento se refiere a una madre de origen árabe (exp.24), que abandona al menor en el parking del Hospital Naval de Cartagena. La noticia fue recogida en la prensa local (La Opinión, 23/11/2004). También, encontramos el caso del abandono de un menor por parte de una madre, ecuatoriana, en un vertedero (exp.15).

\footnotetext{
5 Según datos proporcionados por Salas Martínez existe una alta frecuencia de toxicomanía en los padres biológicos. Según este autor: "el problema de la drogadicción sigue siendo una de las principales causas de desestructuración familiar y la necesidad de que esta población participe en mayor medida en programas de rehabilitación" (Salas Martínez et al., 2009: 40).
} 
Tabla 5. Actividades padres biológicos, 1987-2007

\begin{tabular}{|c|c|c|c|c|c|}
\hline \multicolumn{3}{|c|}{ Madres } & \multicolumn{3}{|c|}{ Padres } \\
\hline Actividad & Frecuencia & $\%$ & Actividad & Frecuencia & $\%$ \\
\hline Limpia escaleras & 1 & 3,6 & Ferrallista & 1 & 2,5 \\
\hline Trabaja en una gasolinera & 1 & 3,6 & Invalido & 1 & 2,5 \\
\hline Lo que encuentra & 2 & 7,1 & Albañil & 1 & 2,5 \\
\hline Trabaja a veces & 1 & 3,6 & Aparcacoches & 1 & 2,5 \\
\hline Club de alterne & 1 & 3,6 & Enlosador & 1 & 2,5 \\
\hline Desempleada & 1 & 3,6 & Obrero/Jornalero & 3 & 7,7 \\
\hline Camarera & 1 & 3,6 & Carpintero & 1 & 2,5 \\
\hline Empleo inestable & 1 & 3,6 & Espartero & 1 & 2,5 \\
\hline Sus labores & 2 & 7,1 & Peón agrícola & 1 & 2,5 \\
\hline Sin profesión & 1 & 3,6 & Chofer & 1 & 2,5 \\
\hline No consta en el expediente & 16 & 57,1 & No consta en el expediente & 23 & 65,7 \\
\hline Total & 28 & 100,0 & Total & 35 & 100,0 \\
\hline
\end{tabular}

Para una mayor claridad en lo comparativo hemos dividido comportamiento, deficiencias y enfermedades y otros problemas en tres bloques. En la Tabla 4 queda de manifiesto, por tanto, que la mayoría de los problemas psicosociales de las mujeres son de comportamiento, llegando a alcanzar el $67,5 \%$ frente al $17,7 \%$ con deficiencias y enfermedades. Respecto a los hombres, los problemas de comportamiento son, igual que en el caso de las madres, mayores $(28,4 \%)$ que los relativos a deficiencias y enfermedades (8,5\%). También destaca, especialmente, un elevado número de padres desconocidos, lo que puede desvirtuar, en cierto sentido, los datos. Se demuestra una menor recogida de información sobre el padre biológico, lo que puede ser debido tanto a su papel secundario como a su desentendimiento y ausencia en el expediente administrativo. Con las consecuentes dificultades para obtener e incluir sus datos.

Otro de los problemas que se encuentran presentes en los padres biológicos es la situación ilegal de algunos de ellos. En las mujeres representa un 14,2\%, mientras que en los hombres un 5,1\%.

\subsection{La actividad profesional de los padres biológicos}

La falta de medios para sustentar a un niño viene también íntimamente relacionada con la profesión, y por lo tanto con el estatus social de los padres. Teniendo en cuenta que el espíritu de las normas de protección de la infancia en la legislación española establece que uno de los principios rectores de la actuación de los poderes pú- blicos es el mantenimiento del niño en el medio familiar de origen, salvo que no sea conveniente para su interés, resulta relevante analizar las actividades que desempeñan las madres y padres biológicos, así como su grado de estabilidad o inestabilidad laboral.

En esta línea, Salas Martínez et al. (2009:40) constatan, tras explorar a familias biológicas, que el nivel educativo de los progenitores de los niños, según los datos que aparecen en los expedientes, son muy escasos. Así, señalan que: en el $77,2 \%$ de los padres y el $72,5 \%$ de las madres, no figura el dato de la educación en los expedientes. Del resto, la mayoría no tenía estudios, tanto en el caso de los padres como de las madres. En cuanto a la situación laboral, el $58 \%$ de los padres y el $83 \%$ de las madres estaban en paro o tenían trabajos ocasionales, lo que sigue poniendo de relieve la problemática social que este colectivo presenta (Salas et al., 2009: 37).

Tras la revisión de los expedientes, de la Consejería de Política Social e Igualdad de Oportunidades, las actividades de los padres biológicos son las que aparecen en la Tabla 5.

La característica más importante de la Tabla 5 es que no consta en la mayoría, ni en los padres ni en las madres, la actividad o profesión. Así, de un 57,1 \% de las madres no sabemos la actividad profesional, llegando a un 65,7 \% en los padres; porcentajes bastante elevados. En el caso de las mujeres, un 21,4 \% representan una clara inestabilidad laboral al utilizar términos como: "Io que encuentra", "trabaja a veces", "desempleada", "empleo inestable" o "sin profesión".

Por el contrario, en el caso de tener un empleo, éstos carecen de un estatus mínimo de cualificación profesional: trabajan en gasolinera, club de alterne, camarera, sus labores o limpiando escaleras (21,4\%). Este último porcentaje es igual al de inestabilidad señalado anteriormente. La consecuencia es que a una cualificación socio-profesional 
de las madres biológicas precaria se une una inestabilidad notable. Incluso el desinterés forma parte de este triangulo socio-profesional. Resulta especialmente gráfico el ejemplo del informe social de 1987:

La madre biológica tiene un completo desinterés y ausencia de cuidado elementales hacía sus hijos, que descarga sus responsabilidades en cualquier otra persona, llegando incluso a obligar, a una mujer mediante la amenaza y el chantaje, de que se encargue del cuidado de sus hijos. Que el trabajo que actualmente ha sido elegido por ella entre otras ofertas de trabajo es el de trabajar en un bar sabiendo que el horario de trabajo sería incompatible con el horario escolar de los niños. (Exp. 2).

El resultado es coincidente y coherente con las características psicosociales y personales reseñadas y analizadas anteriormente. Nos encontramos, por tanto, ante un estatus social que podríamos señalar como, prácticamente, de exclusión.

Respecto a los hombres podemos afirmar que frente a la casi nula actividad de las madres, presentan un estatus socio-profesional de mayor cualificación y estabilidad. Lo cual no quiere decir que la inseguridad ${ }^{6}$, temporalidad y estacionalidad en el trabajo no formen parte de sus vidas cotidianas. Un perfecto ejemplo nos lo muestra el caso de : "Ha trabajado como temporero en la recolección de la aceituna, también suele vender ropa en los mercados como ambulante. Actualmente se dedica a la venta de co$\cos ^{\prime \prime}$ (Exp. 3).

Si llevamos a cabo una división en sectores socio-profesionales de las actividades de los padres, destaca especialmente un 33,3\% dedicado al sector secundario con actividades que requieren una cualificación y formación profesional. Sin embargo, la eventualidad y la inestabilidad forman parte del trabajo cotidiano; tal y como refleja, gráficamente, el siguiente informe de valoración: "Realiza trabajos agrícolas eventuales sin continuidad siendo escasos y mal administrados los ingresos" (Exp.18).

En cuanto al conjunto de las actividades socio-profesionales (Tabla 5) , la mayoría trabaja en el sector primario: albañil, espartero, jornalero, o temporero agrícola (6 casos, $50 \%)$. En el sector secundario hay un total de 4 casos: carpintero, chofer, enlosador o ferrallista; $y$ en el sector terciario únicamente nos encontramos con un padre pensionista y otro sin adscripción socio-profesional que se dedica a aparcar coches.

\subsection{La edad de los progenitores biológicos}

La edad de los progenitores biológicos cuyos hijos, tras el nacimiento, son recogidos o entregados a la Adminis-

\footnotetext{
6 “El padre no tiene trabajo ni profesión determinada". En Informe del asistente social (Exp. 7).
}

tración para su atención, cuidado y protección, refleja una de las diferencias demográficas y socioculturales más notables en comparación con el conjunto de la población. Por otra parte, existen también diferencias entre la edad de las madres y la de los padres biológicos.

El grupo más numeroso de edad de la madre biológica al nacimiento del primer hijo es entre los 17-20 años (11 madres), seguido de 21-24 años (5), y 25-28 años (5) por igual. Los rangos de edad entre 17 y 28 años es el más numeroso de las madres biológicas para tener su primer hijo y darlo en adopción (ver Tabla 6). Otra de las conclusiones a la que podemos llegar es que conforme aumenta la edad de las madres, el número de mujeres que dan en adopción a sus hijos va decreciendo considerablemente.

Tabla 6. Edad de las madres biológicas al primer hijo tenido y entregado/recogido por la Administración, 1987-2007

\begin{tabular}{|c|c|c|}
\hline Edad & Frecuencia & $\%$ \\
\hline$<17$ años & 2 & 7,1 \\
\hline 17-20 años & 11 & 39,2 \\
\hline 21-24 años & 5 & 17,8 \\
\hline 25-28 años & 5 & 17,8 \\
\hline 29-32 años & 1 & 3,5 \\
\hline 33-36 años & 1 & 3,5 \\
\hline 37-40 años & 2 & 7,1 \\
\hline 41-44 años & 1 & 3,5 \\
\hline Total & 28 & 100,0 \\
\hline
\end{tabular}

Sin embargo, la edad media en la primera maternidad en el año 2006 en España es de 32 años, una de las más altas de la Unión Europea, y proviene de una primera maternidad de 28 años en 1996, en el caso de Murcia. Esto demuestra la gran juventud que tienen las madres biológicas en su primer hijo respecto al resto de población en el periodo que estamos estudiando. Lo cual, pone de manifiesto la disparidad tan enorme en Murcia o España respecto de las madres biológicas a las que la entidad pública les retira sus hijos, o ellas lo entregan a la institución para su posterior adopción. Esta realidad demográfica refleja y pone de manifiesto comportamientos extremos y alejados de las medias generales, lo que significa una brecha de desigualdad, exclusión social y marginación. Se comprueba la gran desigualdad y diferencia social entre unas mujeres y otras.

El profundo desfase demuestra la existencia de un grupo social; precisamente el de aquellas mujeres cuyos hijos son entregados o recogidos por la Administración, que se encuentran fuera de los parámetros socioeconómicos y de comportamiento demográfico-cultural del resto de la población. Situación, además, que se confirma al comprobar, en otras tablas, la situación psicosocial o las actividades a 
las que se dedican, y que hemos comentado anteriormente. Incluso, podríamos adelantar, aunque lo analizaremos posteriormente, el rechazo y obstrucción generalizada por parte de las familias adoptantes a mantener contacto o permitir que los padres biológicos, especialmente la madre -el padre suele abandonar cualquier preocupación sobre el hijo procreado en situación de irregularidad social-, se relacionen con el hijo, finalmente adoptado. Otro aspecto relacionado con la edad de las madres biológicas es la edad que tienen en el último hijo entregado/retirado cuando ya ha tenido dos o más hijos, como se recoge en la Tabla 7. Debemos de considerar que pueden existir más hijos que, posteriormente, la madre haya podido concebir y que desconozcamos su existencia. En general son muy jóvenes al último hijo -a excepción de 4 casos de: 30 años (1), 34 años (2) y 40 años (1)- en 9 sobre 13 casos (69,2\%) no superan los 23 años.

La indicación de la edad de los padres es muy escasa, solo conocemos la de catorce de un total de treinta y cinco; mientras que en el caso de las madres, como ya hemos comprobado, el porcentaje de datos se eleva a veintiocho. Al igual que ocurre en otros indicadores, las informaciones recogidas por los técnicos de la entidad pública inciden mucho más en el caso de las madres que en el de los padres.

Tabla 7. Edad de la madre biológica al último hijo 1987-2007

\begin{tabular}{|c|c|}
\hline Edad al último hijo recogido & Número de madres \\
\hline 19 & 1 \\
\hline 20 & 3 \\
\hline 22 & 4 \\
\hline 23 & 1 \\
\hline 30 & 1 \\
\hline 34 & 2 \\
\hline 40 & 1 \\
\hline
\end{tabular}

En cuanto a la edad del padre al primer hijo tenido, presenta un ritmo distinto. Así, solamente $3(21,4 \%)$, tienen entre 17 y 20 años. Recordemos que en el caso de las madres alcanzaba en este mismo intervalo el 39,2 \%. Lo que confirma una clara disfunción entre hombre-mujer ${ }^{7}$. El $42,8 \%$ (6 casos de un total de 14) no supera los 24 años. Sin embargo, en el caso de la mujer, un 64,1 \% (18 casos sobre 28), tienen 24 años al primer hijo (véase Tabla 6).

\footnotetext{
${ }^{7}$ Recordemos que más de la mitad de los padres son desconocidos, y no consta ninguna información sobre ellos.
}

Tabla 8. Edad del padre biológico al primer hijo recogido o entregado a la administración, 1987-2007

\begin{tabular}{|c|c|r|}
\hline Edad (años) & Frecuencia & $\%$ \\
\hline $17-20$ & 3 & 21,4 \\
\hline $21-24$ & 3 & 21,4 \\
\hline $25-28$ & 1 & 7,1 \\
\hline $29-32$ & 2 & 14,2 \\
\hline $33-36$ & 0 & 0,0 \\
\hline $37-40$ & 1 & 7,1 \\
\hline $41-44$ & 2 & 14,2 \\
\hline $45-48$ & 0 & 0,0 \\
\hline $49-53$ & 2 & 14,2 \\
\hline Total & 14 & 100,0 \\
\hline
\end{tabular}

\subsection{Origen y estado civil}

Según los expedientes consultados, un muy elevado porcentaje de madres biológicas $(67,8 \%)$ son de origen español. Y todas de Murcia, a excepción de seis que nacieron en Almería, Asturias, Logroño, Barcelona, Jaén y Castellón, pero domiciliadas en la Región de Murcia. Es a partir de 1998 cuando comienzan a entregar o recogerles hijos a mujeres procedentes de otros países: Ecuador (4), Rumanía (1), Rusia (1), Bulgaria (1) o países árabes (3), con un predominio de madres sudamericanas. Murcia en el año 2001 será una de las Comunidades Autónomas con mayor número de ecuatorianos empadronados. En 2006 alcanza una cifra total de 52.727, de las más elevadas de toda España $(3,72 \%)$. Lo que es debido al boom migratorio durante los últimos años de la década de los noventa. El porcentaje de madres extranjeras llega al 31,8\%.

En cuanto al origen de los padres, 12 son de nacionalidad española y 2 árabes. Sin embargo, esta variable puede estar desvirtuada por el elevado número de padres no conocidos (21). Este desconocimiento incide, igualmente, respecto al estado civil de los padres, ya que del total de padres conocidos: 6 son solteros, 5 casados y 3 separados. Así, aunque existan hombres casados, la mayoría serán solteros en el momento de la retirada o entrega del menor. La conclusión es que pese a la elevada diferencia entre madres solteras (75 \%) y padres solteros $(17,1 \%)$, el desconocimiento de 21 padres sobre un total de 35 (60\%), no nos permite plantear conclusiones definitivas. De todas formas, se produce una fuerte presencia de madres solteras, (21) respecto a 6 casadas y 1 separada. Lo que significa un predominio notable de aquellas (75\%), que contrasta, aunque dentro de la misma tendencia, con mayoría de padres solteros: 6 , pero divididos por la suma de casados y separados: 8.

En un estudio realizado (Salvatierra, 2003:2) sobre procesos de adopción entre 1977 y 1992, en Lisboa, el 64 \% 
de las madres biológicas eran solteras, el 18,6 \% desconocidas, el 8,8 \% casadas, el 6,8 \% divorciadas o separadas, y el $1,8 \%$ viudas. En el caso español sucede lo mismo en tanto en cuanto la mayoría de las mujeres son solteras a la fecha de dar en adopción a sus hijos biológicos. La mayoría de las madres biológicas son españolas y solteras. A partir de 1998, las mujeres proceden de otros países, como, por ejemplo, Ecuador o Marruecos. Los padres son también españoles, aunque más separados que solteros. En este sentido, podemos deducir la promiscuidad de estos hombres.

¿Cuáles son las causas de este distinto comportamiento padre-madre? Una primera explicación colocaría a las características psicosociales de las madres biológicas, por su situación de exclusión, como mujeres solteras en su mayoría. En el caso de los hombres puede deberse a que tengan relaciones extramatrimoniales, lo cuál explicaría un número de casados y separados (8) superior al de solteros (6). Si esta tendencia se cumpliese en el muy elevado número de desconocidos (21), se confirmaría esta explicación.

Las características, edad y estado civil se convierten en diferencia de género. El estado civil se suma a la edad, con lo que los comportamientos y las prácticas distancian y separan hombre-mujer. Se pone de manifiesto la ausencia de sentido y sentimiento de pareja o unidad familiar. Es muy débil, o casi nula.

4.5. Integración del menor en su familia biológica. Casuistica

El objetivo prioritario y fundamental de la administración y equipos técnicos que trabajan en la sección de acogimiento y adopción es lograr la incorporación de los menores en su familia natural para recuperar sus señas de identidad y una existencia y vida en ambientes que no presenten riesgos.

En este punto se plantea el debate sobre si es realmente beneficioso para los menores el regreso a sus familias de origen o, por el contrario, sería preferible, a pesar de la mejora en las condiciones, crecer en una familia que le garantice unos niveles mínimos de bienestar. Entramos en el debate jurídico, apuntado anteriormente, del interés del menor.

Las entidades públicas intentan recuperar las condiciones óptimas de la familia biológica y, especialmente, de la madre (en muchas ocasiones el padre es desconocido); es decir, otorgar un periodo de tiempo para que se produzcan modificaciones de conducta ${ }^{8}$. La Ley establece un plazo de dos años desde la notificación de la resolución de la declaración de desamparo, para el caso de que la Entidad Pública pueda adoptar cualquier medida, incluida la propuesta de adopción, cuando exista un pronóstico fundado

\footnotetext{
${ }^{8}$ Artículo 172.3 Código Civil: "La Entidad Pública, de oficio o a instancia del Ministerio Fiscal o de persona o entidad interesada, podrá revocar la declaración de situación de desamparo y decidir el retorno del menor con su familia, siempre que se entienda que es lo más adecuado para su interés. Dicha decisión se notificará al Ministerio Fiscal".
}

de imposibilidad de retorno a la familia de origen (artículo 172.2 in fine Código Civil).

Cuando estas mujeres no acuden a reuniones orientativas, no visitan a su/s hijo/s en el Centro dónde se encuentran internado/s o presentan, incluso, conductas agresivas y violentas hacia los equipos técnicos además de una clara desmotivación y despreocupación por saber en qué condiciones se encuentran su/s hijo/s en los Centros de Acogida, los equipos empiezan a valorar la implantación de otras medidas de protección.

Cuestión distinta será al producirse el arrepentimiento de las madres e iniciar éstas procesos judiciales sobre la decisión tomada por parte de la Administración Pública de llevar a cabo el proceso de adopción, si es que eso fuera lo que finalmente ésta decide. Así, una noticia publicada en abril de 2015 en el diario La Verdad (Murcia) explica la denegación de la patria potestad por parte de la Audiencia Provincial a una mujer que reclamó sus hijos dados en adopción. Alegaba que los niños vivían en la pobreza pero que no estaban desamparados, tal y como habían apuntado los técnicos en adopción de la Administración. La Sala expuso que la apelante no impugnó en su día la declaración de desamparo, como tampoco recurrió las medidas de acogimiento preadoptivo sin visitas. No asistir a la vista principal del juicio donde se jugaba la posibilidad de recuperar a sus hijos, se convirtió en la prueba objetiva que indicaba desinterés y falta de responsabilidad ( La Verdad, 14/04/2015).

La madre, en el expediente de adopción, siempre tiene que ser oída para constatar si, efectivamente, y debido a sus circunstancias personales resulta aconsejable la retirada o no de la patria potestad de sus hijos. En la mayoría de las ocasiones, la conducta de la madre biológica es el resultado de un desinterés en la custodia de sus propios hijos. Así, se refleja en uno de los expedientes analizados: la madre no acude regularmente a las visitas que tiene autorizadas en el Centro de internamiento de su hijo/a. Los técnicos constatan la falta de asiduidad de la madre biológica a las visitas autorizadas mediante partes de firmas de las personas encargadas del Centro?.

Debido a estos problemas y al dramatismo de sus situaciones, entendemos el temor de los futuros padres adoptivos ante posibles comportamientos y conductas que podrían ser agresivas por parte de las madres biológicas. Y que resultan para ellos inquietantes, preocupantes y nada positivas para la estabilidad de la nueva unidad familiar $y$, especialmente, del menor. En este sentido, nos encontramos comportamientos como, por ejemplo, la interposición de recursos ante el Juzgado correspondiente cuando ya se ha producido en firme la adopción; conductas agresivas hacia los adoptantes o la vigilancia y seguimiento de una manera obsesiva por parte de la familia biológica cuando sus hijos se encuentran con la nueva familia o, lo que sucede a veces, también con la familia extensa del menor. Incluso se producen, en ocasiones, manifestaciones violentas con los técnicos del Servicio de Adopción de las entidades públicas $^{10}$.

\footnotetext{
9 Entre los diferentes ejemplos que podríamos incluir, señalamos el Exp. 23/2003-2009.

${ }^{10}$ Exp. 1, 1987-1989. Los abuelos se personan el 25/04/88, en el Negociado de
} 
Tabla 9. Ejemplos en los que la familia biológica presenta recurso ante los órganos judiciales, 1987-2007

\begin{tabular}{|c|c|c|c|c|}
\hline № Expediente & Quien lo presenta & $\begin{array}{c}\text { Fecha de pre- } \\
\text { sentación }\end{array}$ & Recurso & Resultado/fecha \\
\hline 2 & Padre biológico & 22-11- 1990 & Oposición al acogimiento & Se desestima (14-05-1991) \\
\hline \multirow{5}{*}{5} & \multirow{5}{*}{ Progenitores biológicos } & 8-04-2002 & $\begin{array}{l}\text { Recurso de apelación con- } \\
\text { tra auto de acogimiento }\end{array}$ & $\begin{array}{l}\text { Se declara desierto (25-07- } \\
\text { 2002) }\end{array}$ \\
\hline & & $4-07-2003$ & $\begin{array}{l}\text { Recurso apelación contra la } \\
\text { adopción }\end{array}$ & Se desestima (20-04-2004) \\
\hline & & $20-11-2003$ & $\begin{array}{l}\text { Sentencia (necesario con- } \\
\text { sentimiento padres) }\end{array}$ & \\
\hline & & 7-01-2004 & Recurso de apelación & \\
\hline & & 20-04-2004 & $\begin{array}{l}\text { Se revoca la sentencia (pa- } \\
\text { dres sean solo oídos) }\end{array}$ & Se desestima (24-06-2004) \\
\hline 11 & Madre biológica & & $\begin{array}{l}\text { Oposición de la madre a la } \\
\text { adopción }\end{array}$ & $\begin{array}{l}\text { Archivo de la demanda } \\
(27-04-2004)\end{array}$ \\
\hline 12 & Progenitores biológicos & 13-12-1999 & $\begin{array}{l}\text { Recurso de apelación con- } \\
\text { tra tutela }\end{array}$ & $\begin{array}{l}\text { Se desestima }(20-03-2000) \\
(12-09-2002)\end{array}$ \\
\hline
\end{tabular}

Fuente: elaboración propia.

En este sentido, las madres biológicas recurren a la justicia para recuperar o alegar que tienen derecho a la patria potestad de sus hijos. Pero sus recursos son siempre desestimados, casi todos, y se ratifica la adopción de los menores. Esta afirmación se contradice con un caso excepcional del que se hizo eco toda la prensa nacional y regional durante el mes de septiembre de 2016 (La Verdad, 12/09/2016 y 13/09/2016). Una pareja de Valencia había tenido que entregar por orden judicial a su hijo preadoptivo a la madre biológica (La Verdad, 12/09/2016) ${ }^{11}$; al día siguiente la prensa recogía en titular la declaración de la madre biológica: "Yo no les di al niño en acogida. A mí me lo arrebataron". El niño tenía cuatro años y permanecía con el matrimonio de acogimiento desde los 18 meses de edad, finalmente tuvo que ser entregado a la madre biológica, de 19 años, y nacida en Guinea Ecuatorial. Así, lo dictaminó la Sentencia de la AP Sección n4 de Oviedo de 10 de marzo de 2016, en la que estima el recurso de apelación interpuesto por la madre biológica contra la sentencia dictada por el Juez de Primera Instancia n7 de Oviedo, en donde se desestimaba la oposición presentada por la madre natural contra la Consejería de Bienestar Social y Vivienda, declarando no preceptivo el asentimiento de la madre en la adopción de su hijo biológico por hallarse ésta incursa en causa de privación de la patria potestad.

Para la Sala de la AP de Oviedo, M.J., la madre biológica no se encuentra incursa en causa de privación de patria potestad. Sigue diciendo que la Administración, desde el mismo día del parto, decidió separar a la madre del recién nacido sin que se barajaran otras opciones que posibilitaran o propiciaran la relación madre/hijo. Más aún, cuando la institución debe de proporcionar, si no sabes, y eres menor de edad (como sucedía en el caso de la madre bioló-

Protección y Tutela diciendo que no están de acuerdo con la medida adoptada por el ISSORM de entregar al menor a una familia acogedora y dicen: "Sentencia tiene usted de muerte, no salga usted a la calle".

11 Aparecía con el titular: “Una pareja de Valencia entrega por orden judicial a su hijo preadoptivo a la madre biológica" gica que contaba con la edad de 15 años cuando da a luz a su hijo, y a su vez, se encontraba tutelada por la entidad pública), como afrontar una maternidad. Y es más que sorprendente que, a pesar de haber solicitado ayuda, no se le han procurado los medios necesarios que insistentemente dice la Administración que le faltan. Según informe del psicólogo sobre la falta de actitud y de aptitud, y teniendo en cuenta la edad de M.J., resulta lógico la ausencia de la capacidad en atención a las especiales circunstancias; pero "no son base objetiva suficiente para denegar o frustrar la relación madre/hijo. Esta niña de 15 años no muestra rechazo hacia su hijo aunque sí inexperiencia, cosa muy distinta, a nuestro parecer" y según sentencia de la AP. Por otro lado, se realizan pruebas periciales (dos psicólogos y trabajadores sociales) que constatan que la madre biológica no presenta deficiencia para el ejercicio de la maternidad responsable, y que por tanto no es incapacitante para ejercer la patria potestad del menor.

Desde la sentencia de fecha de 10 de marzo de 2016 hasta que se hace efectiva la entrega del menor a la madre biológica, transcurren 6 meses. En este periodo de tiempo se produce la búsqueda de los padres de acogida por las fuerzas de seguridad para el cumplimiento de la condena, ya que los acogedores se encontraban oficialmente como" desaparecidos". Finalmente, el 12 de septiembre de 2016 la madre biológica recupera a su hijo, entregado por la familia, y dejando sin efecto el acogimiento familiar preadoptivo del menor.

Como se aprecia en la Tabla 9, recogemos ejemplos de familias biológicas que presentan recursos de oposición contra el auto de acogimiento y adopción; en uno de los casos el padre biológico, en otro la madre biológica, y en otros dos casos ambos progenitores.

En ocasiones, las madres biológicas reclaman a sus hijos diciendo que se encuentran en condiciones adecuadas para cuidarlos. Consideramos que también existe cierta impotencia por parte de las madres que por una causa $u$ otra no pueden hacerse cargo de ellos. Podemos afirmar 
que no solamente existen casos de irresponsabilidad, o por lo menos, no debemos de generalizar en este aspecto, sino tener una visión más amplia y grandes dudas de que haya rehecho su forma de vida y se encuentre en condiciones, aunque sean muy modestas, de ofrecer un hogar adecuado a su hijo/a biológico. La prensa nacional ${ }^{12}$ se ha hecho eco de algunos ejemplos que demuestran la reclamación de las madres biológicas para recuperar la patria potestad de sus hijos.

En los círculos más cercanos a la adopción existe un pensamiento generalizado de que las madres biológicas son más que problemáticas e incluso: "no capacitadas para mantener una familia".

Cuando se realizó una entrevista a un padre adoptante expresó:

No quiero que mi hijo adoptado mantenga contacto con la madre biológica. Optamos por la modalidad internacional porque teníamos miedo a la madre biológica y a los posibles recursos que pudiera poner en los Juzgados (Entrevista, 20/03/2015).

Otra madre soltera, y adoptante declara que ella "quería que la adopción nacional fuera una renuncia de la madre y no una retirada por parte de la Administración" (Entrevista, 09/02/2015).

Una de las madres adoptivas expresa el miedo que tiene de que su hijo adoptado pueda tener contacto con su madre biológica:

Pues claro que tengo miedo, cómo no voy a tener miedo? Tengo miedo de que no pueda hacer nada porque es mi hijo pero tengo miedo de complicarme la vida. [...] yo en la idoneidad tuve que poner que si consentiría que mi hijo tuviera contacto con su familia biológica, pero siendo menor NO. Estoy viendo casos en la asociación de niños de nacional de 15 años o de 12 que están pidiendo ver a sus padres. No tienen 18 pero los padres lo han consentido, han sacado todo el informe y están buscando a la madre (Entrevista, 23/06/2015).

Con estos ejemplos, se observa el temor de los futuros candidatos a que los niños puedan ser recuperados por la familia biológica.

\section{Conclusiones}

Tras haber realizado nuestro recorrido por el estudio socio-demográfico de las familias biológicas en la Región de Murcia a lo largo de la coyuntura 1987-2007, consideramos que, en perspectiva comparada, faltan a escala nacional análisis bibliográficos (al haberle otorgado prioridad a factores psicológicos y de comportamiento) sobre las problemáticas sociales de las familias biológicas.

\footnotetext{
12 El País (13-09-2016; 14-09-2016 y 22-09-2016); La Opinión (12-09-2016); La Verdad (14-09-2016).
}

Los resultados de la investigación permiten plantear que la edad de las madres biológicas a la primera maternidad es muy temprana: de 17 a 20 años; el grupo: 21 a 24 años, es el segundo más numeroso. Las medias generales de España en el mismo periodo de tiempo son muy distintas y, sobre todo, mucho más elevadas. Lo que significa una realización personal o/y de pareja, unido a un proyecto de vida, del que los protagonistas de estas situaciones, carecen. Por tanto, estamos ante un sector de la población de exclusión social y de marginación. En el caso de los padres, desconocemos la edad de la mayoría; según los datos que tenemos, el primer periodo de edad comprende de los 17 a 20 años, y de 21 a 24 años en segundo lugar. Así, pues, se produce coincidencia en la edad de la madre y del padre.

Igualmente, es de destacar que los técnicos de los Servicios de Adopción dan más importancia a la madre biológica que al padre biológico. En las tablas que hemos elaborado de la familia de origen, muchos datos del padre son desconocidos, y así se aprecia igualmente en los documentos elaborados por los profesionales del Servicio en los que se da más información de la madre que del padre. Ciertamente, esta cuestión responde a la naturaleza biológica de la maternidad, pero también hay que destacar la necesidad de realizar estudios que aborden esta cuestión desde la perspectiva de género, en la que se dilucide la problemática al respecto de la masculinidad.

En cuanto a los datos que posibilitan una mínima cuantificación, los factores psicosociales del padre y de la madre biológica reflejan que los problemas más comunes en ambos son de comportamiento (irresponsabilidad, dejadez, inmadurez). Se produce la ruptura de un tópico, ya que existe una opinión generalizada de que la madre biológica presenta una elevada fecundidad cuando realmente no es así. Sin embargo, debemos de señalar que existen en nuestro estudio casos de un elevado número de hijos biológicos, incluso con otros hombres, o niños que no aparecen en los expedientes y de los cuales no sabemos nada.

Respecto a la actividad laboral de los padres, suelen constar los de las madres: mujeres con mucha inestabilidad laboral; y en el caso de los hombres (en la mayoría de los casos estudiados no se indica en el expediente), presentan una mayor cualificación y estabilidad pero, también, con una clara eventualidad en la actividad que realizan.

Se observa que son familias de bajo estatus social, y con unas dificultades tales que obligan, por tanto, a la Administración Pública a llevar a cabo la retirada de sus hijos biológicos. Aunque el espíritu de la norma sea que el menor vuelva a su familia de origen, en ocasiones, esto no es posible, y se pondría en marcha la adopción de alguna medida de protección a la infancia. Estamos, pues, ante una brecha social de marginación y de desigualdad, como señalamos al analizar la edad de los progenitores. Una de sus consecuencias será el abandono de los hijos y la necesidad y obligación, por parte de las instituciones públicas (en defensa y protección del menor), de retirarles los hijos y entregarlos al cuidado y atención de la Administración. Pero el deseo de los padres adoptantes, casi siempre, es a romper cualquier vínculo del menor con su familia de origen. Sin embargo, esto entraría en contradicción con la norma jurídica (artículo 178 Código Civil) ya que permite que cuando el interés del menor así lo aconseje puede 
acordarse el mantenimiento de alguna forma de relación o contacto a través de visitas o comunicaciones entre el menor, los miembros de la familia de origen y la adoptiva. Será, por tanto, el juez, al constituir la adopción, quién podrá acordar el mantenimiento de la relación, determinando su periodicidad, duración y condiciones, a propuesta de la Entidad Pública o el Ministerio Fiscal, y con el consentimiento de la familia adoptiva y del adoptando si tuviera suficiente madurez $y$, siempre, si fuere mayor de doce años. El Juez también puede acordar la modificación de dicha medida o la finalización en atención al interés superior del menor.

Nos encontramos, como hemos apuntado anteriormente, en una contradicción entre los deseos de la familia adoptiva y el espíritu normativo. E insistiríamos, para concluir, que debemos tener una visión más amplia de los problemas de las madres biológicas: la falta de recursos económicos y la situación de inestabilidad vital y emocional es posible que les obligue a dar en adopción a sus hijos con el consiguiente sentimiento de pérdida emocional que ello supone. Por otra parte qué duda cabe que existe un prejuicio hacia estas madres respecto a su vida anterior.

En conclusión, podríamos afirmar que si jurídicamente se ha avanzado, socialmente nos encontramos en una fase embrionaria para alcanzar la propuesta del legislador. Propuesta, además, muy necesitada de aplicar políticas públicas que disminuyan las desigualdades sociales, verdadera razón y causa última del desfase entre la realidad social de las familias o/y madres biológicas y la normativa legal. En esta ocasión, la ley supera a la evolución social.
Fuentes y bibliografía

Fuentes legislativas

Código Civil Español (BOE n 206 de 25 de julio de 1889)

Ley Orgánica 8/2021, de 4 de junio, de protección integral a la infancia y la adolescencia frente a la violencia (BOE $n^{\circ} 134,05 / 06 / 2021$ ).

Bibliografía

CHACÓN MARTÍNEZ, Ana (2019): El interés superior del menor. Historia de un reconocimiento jurídico en los derechos humanos para la infancia (siglos XVIII-XXI). Murcia, Editum.

FARGE, A. (1991): La atracción del archivo. Valencia, Edicions Alfonsa El Magnánim.

FERNÁNDEZ MOLINA, Milagros (2004): “Los estudios españoles sobre adopción y acogimiento familiar 1974-2004", Boletín de Psicología, 81, pp. 7-31.

MARRE, D. y BESTARD, J. (2004): "Sobre la adopción y otras formas de constituir familias: a modo de introducción", en D. Mare y J. Bestard (eds.), La adopción y el acogimiento: presente y perspectivas. BarceIona, Estudis d'Antropologia Social i Cultural. Universidad de BarceIona, pp. 13-72

MAYOR DEL HOYO, M V. (2020): “El asentimiento a la adopción de la madre biológica y la limitación de la autonomía de la voluntad de la mujer. Una propuesta de lege ferenda", Revista de derecho privado, 104, pp. 105-122.

SALAS MARTINEZ, M.; FUENTES REBOLLO, M. J.; BERNEDO, I. M.; GARCÍA MARTÍN, M.A. y CAMACHO PÉREZ, S. (2009): "Acogimiento en familia ajena y visita de los menores con sus padres biológicos", Escritos de Psicología, 2 (2), pp. 35-42.

SALVATIERRA, F. (2003): "A adopcao no melhor interesse da crianca", Revista da Santa Casa da Misericórdia de Lisboa, Solidária Cidade,10, pp, 1-36.

REYES LEGAZA, C. y CANTERA REY, C. (2021): “La familia biológica en el acogimiento familiar. Pistas para su evaluación e intervención", Itinerarios de Trabajo Social, 1, pp. 71-78. 